\title{
Biotechnical System for Recording Phonocardiography
}

\author{
Marwan Ahmed Ahmed Hamid ${ }^{1}$ \\ Dept. of Biomedical and Food Engineering \\ NED University of Engineering and Technology \\ Karachi, Pakistan
}

\author{
Maria Abdullah ${ }^{2}$ \\ Dept. of Computer System Engineering, Dawood University \\ of Engineering and Technology, Karachi, Pakistan
}

\author{
Najeed Ahmed Khan ${ }^{3}$ \\ Dept. of Computer Sc. and Info. Technology \\ NED University of Engineering andTechnology \\ Karachi, Pakistan
}

\author{
Yasmin Mohammed Ahmed AL-Zoom ${ }^{4}$ \\ Dept. of science and Technology \\ IBB University, Ibb, Yemen
}

\begin{abstract}
The Phonocardiography is a graphical method of recording of the tones and noise generated by the heart with the help of the phonocardiogram machine. Cardiovascular disease (CVD) and heart failure (HF) are considered life-threatening and mostly cause death. The phonocardiograph signal (PCG) considers an indicator of abnormalities in the cardiovascular system. It provides the ability to carry out qualitative information and quantitative analysis of different tones and heart murmurs. PCG plays a major role in treatment, diagnosis and decision making of the clinical examination and biomedical research fields. The use of simple stethoscope for diagnosis the heart problem requires an experienced physician or doctors. Many people with CVD and HF are dying every day because of the lack of facilities that analysis the heart defects. Most of the low come countries suffer from a severe shortage in the Electrocardiogram (ECG) and PCG devices and trained physicians and doctors. The Poor healthcare system in these countries needs to be improved especially the problem of heart disease diagnostics. The PCG is a technique for recording and monitoring the cardiac acoustics by using a transducer and microphone. This paper attempts to design a cheap and simple biotechnical system for recording and monitoring PCG signal. The hardware was designed and implemented using the stethoscope, electret microphone, amplifiers, DC source, and jack for transmitting the PCG signal to the computer. The software was codded for monitoring and processing the PCG signal.
\end{abstract}

Keywords-Phonocardiograph (PCG); cardiovascular diseases (CVD); heart failure (HF); electrocardiograph (ECG)

\section{INTRODUCTION}

The phonocardiography method is a non-invasive investigation method that is recommended to evaluate the HF and considered as an essential tool for diagnosing CVD [1]. It provides crucial information about several heart problems.

These days the PCG signal is less used because of the wide and effective use of the ECG signal and echocardiography, but still many cardiac defects are best diagnosed by the PCG [2]. The anatomy of the human heart is described as four chambers (two auricles and two ventricles) for collection and pumping out of the blood, respectively [3]. The heart beating (sound) is described as a complex interaction among pressure components, contraction of the heart chambers (muscles) and blood vessels flow [4]. The mechanical process of contraction of the heart muscles and the flow of the blood result in vibrations and acoustics that can be recorded over the chest [5].

The PCG is one of the most effective ways to evaluate heat function. Wang Haibin et al. [6] developed a system for inhome use of heart abnormality monitoring. Their system can auscultate respiratory sounds, heart sounds and lung sounds. Therefore, these sounds contain a lot of valuable information for the diagnosis of heart problems.

Recently, with rapid growth of computer hardware and digital signal processing methods, PCG signal could be recorded and analyzed [7]. A. Atbi el al [8] presented an algorithm for detection of the heart sound. Their method based on remove the heart noise by using the low pass filters and also detected the S1 and S2 peaks of the heart sound.

The recorded sound of the heart (PCG) can be defined as the main (S1 and S2) and sub main (S3 and S4) components [9]. Fig. 1 shows the all classes of PCG components whereas Fig. 2 shows location of S-S interval of PCG and R-R interval of ECG.

The diastolic segment (S1and S2) is important in cardiovascular diagnosis. The Diastolic part of the PCG signal studied Metin Akay et al. [10] they identified the S1 as the loudest class of PCG signal and it happens during ventricular contraction. The S1 class contains a series of low frequency vibrations ranging between $25 \mathrm{~Hz}$ and higher amplitudes with time terms of $100 \mathrm{~ms}$ to $200 \mathrm{~ms}$. The S2 class appears at the end of ventricular systole for about $0.12 \mathrm{~s}$ with a frequency of $50 \mathrm{~Hz}$, which is higher in frequency, but shorter in time terms than S2 [11]. The third class (S3) can be heard at the beginning of diastole, while a class S4 in the PCG signal [12].

The improvement of the biomedical engineering technology has led to renew some of the useful medical detection methods. Noor Kamal et al. [13] introduce new approach to detect specific measurements that relate to biomedical signal such as PCG and ECG. In their result, the first and second heart sound (S1, S2) can be determined from another signal like ECG. 

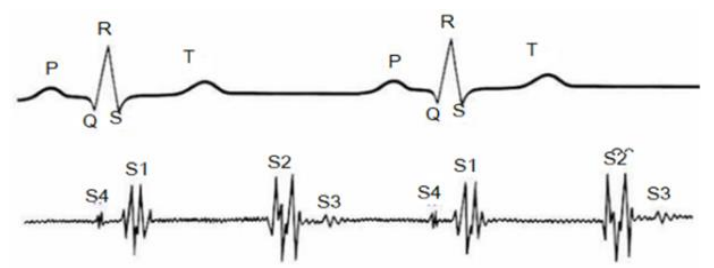

Fig. 1. All Classes (S1, S2, S3, and S4) of the PCG Signal.

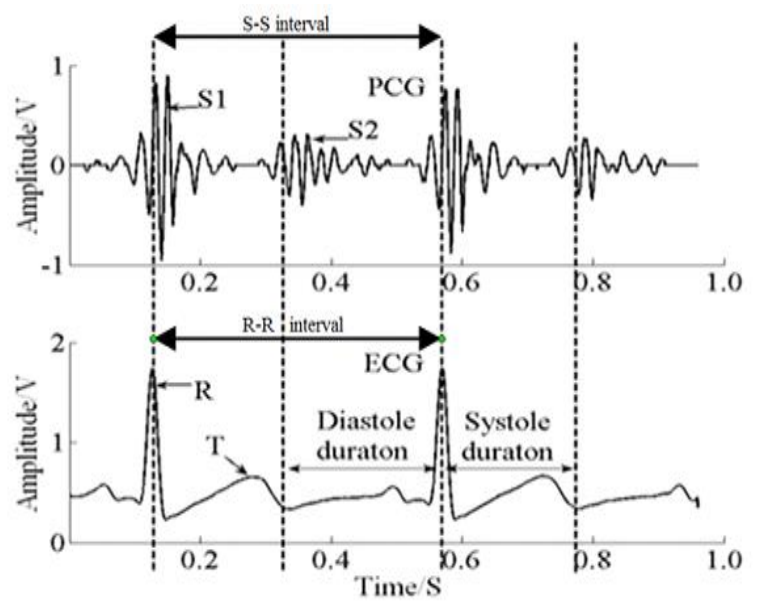

Fig. 2. Main Components of Normal PCG Signal with Location of S-S Interval of PCG and R-R Interval of ECG.

The rate of cardiovascular diseases with each passing day is alarming [14] especially in poor countries. The importance of the phonocardiography can be identified from the simplicity of this technique and also from the amount of information that can have from PCG signal. The doctors and physicians mostly use the heart sound to evaluate the different heart functions and detect the abnormalities of the heart. The shortage of the medical diagnostic equipment is the main reason for the poor diagnostics and then treatment of the heart diseases. The following methodology has been employed to overcome the problem of the heart medical diagnostics by developing an efficient and uncostly biotechnical system for recording and storing the PCG signal.

\section{Proposed Methodology}

Auscultation of the heart is a method of listening to the sound of the heart and can be recorded from specific points on the chest. In medical diagnostics, the PCG signal is collected from the S1, S2, S3, and S4 components over the chest as demonstrated in Fig. 3.

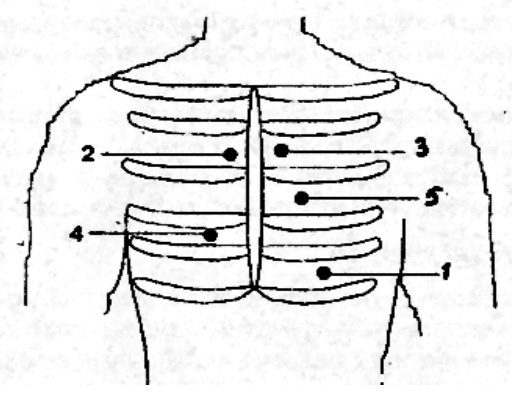

Fig. 3. Heart Auscultation Points.
In this paper we proposed a biotechnical system that may be a modified stethoscope for recording the PCG signal. In proposed biotechnical system the earpieces of the stethoscope are replaced by an electret (sensor (CZN-15E) microphones. The output from the sensors is amplified by amplifiers (NE5534P). The jack connector fed the amplified signal and then fed into PC system. The recoded PCG signal undergoes several steps of transmitting the sound into an electrical signal, then the electrical signal is amplified and converted to a digital signal [15]. The signal process is preformed to denoise and improve the quality of the PCG signal. The software has a friendly graphical user interface (GUI) for monitoring the PCG signal [16]. The data and metadata can be stored in digital form. The proposed biotechnical system contains the following parts.

\section{A. Selection of Components and Circuit Design}

The selection of appropriate elements for any circuit is important to get an accurate and efficient result. The selection of the stethoscope (Fig. 4) is not much important, as the selection of an electret microphone Fig. 5. The electret microphone is a type of capacitor microphone that does not need a power supply for polarizing voltage [17]. The proposed electret microphone in this method is a sensor type of CZN$15 \mathrm{E}$. Table I presents the characteristics of the required electret microphone (CZN-15E).

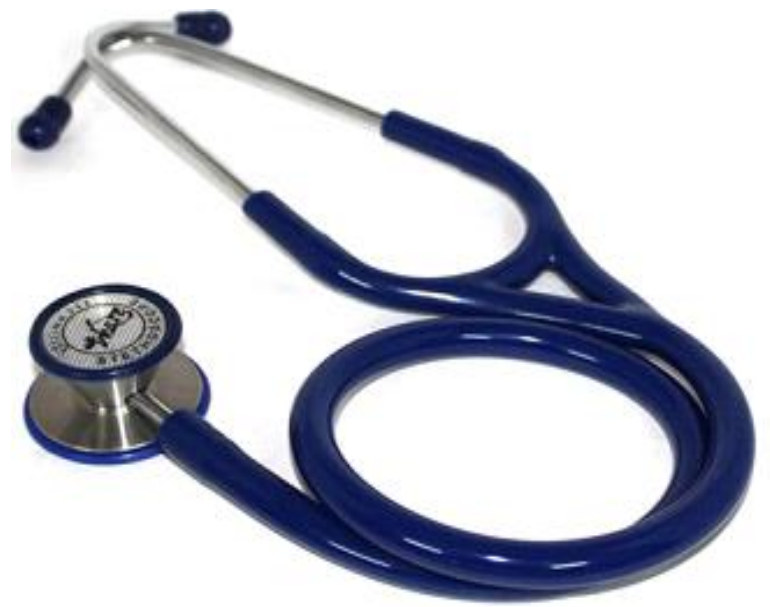

Fig. 4. Stethoscope.

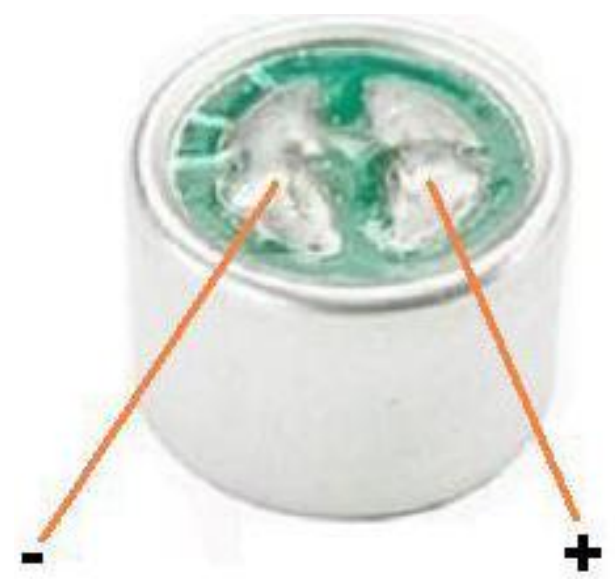

Fig. 5. Electret Microphone. 
TABLE. I. CHARACTERISTIC OF THE SELECTED ELECTRET MicRoPHONE $(\mathrm{CZN}-15 \mathrm{E})$

\begin{tabular}{|l|l|}
\hline Feature & Description \\
\hline Sensitivity & $-58 \pm 2 \mathrm{~dB}(0 \mathrm{~dB}=1 \mathrm{~V} / \mathrm{pa}, 1 \mathrm{KHz})$ \\
\hline Impedance & Low impedance \\
\hline Directivity & Omnidirectional \\
\hline Frequency & $20-16,000 \mathrm{~Hz}$ \\
\hline Voltage range & $1.5 \mathrm{~V}-10 \mathrm{~V}$ \\
\hline Standard operation voltage & $4.5 \mathrm{~V}$ \\
\hline Current consumption & Max.0.5mA \\
\hline Sensitivity reduction & Within $-3 \mathrm{~dB}$ at $3 \mathrm{~V}$ \\
\hline S/N ratio & More than $60 \mathrm{~dB}$ \\
\hline
\end{tabular}

The heart sound hits the diaphragm by vibrated air particles, as a result, the diaphragm changes the distance between the plates. The electret substance moves on the backplate, producing a voltage across the capacitor [18]. The produced voltage is weak and needs to be fed to the amplifier. The required amplifier should be low noise with high-speed audio. The implemented operational amplifier in this method is NE5534P [19]. Table II contains the main features of the required amplifier (NE5543P).

The electrical circuit diagram was designed via any appropriate circuit design software (Multisim [20]). Fig. 6 shows the biotechnical circuit of the proposed system.

TABLE. II. CHARACTERISTIC OF THE SELECTED AMPLIFIER (NE5534P)

\begin{tabular}{|l|l|}
\hline Feature & Description \\
\hline Equivalent Input Noise Voltage & $3.5 \mathrm{nV} / \sqrt{ } \mathrm{Hz}$ Typ \\
\hline Unity-Gain Bandwidth & $10 \mathrm{MHz}$ Typ \\
\hline Common-Mode Rejection Ratio & $100 \mathrm{~dB}$ Typ \\
\hline High DC Voltage Gain & $100 \mathrm{~V} / \mathrm{mV}$ Typ \\
\hline Peak-to-Peak Output Voltage Swing & $\begin{array}{l}32 \mathrm{~V} \text { Typ with } \mathrm{VCC} \pm= \pm 18 \mathrm{~V} \text { and } \\
\mathrm{RL}=600 \Omega\end{array}$ \\
\hline High Slew Rate & $13 \mathrm{~V} / \mu \mathrm{s}$ Typ \\
\hline Wide Supply-Voltage Range & $\pm 3 \mathrm{~V}$ to $\pm 20 \mathrm{~V}$ \\
\hline Low Harmonic Distortion & \\
\hline Offset Nulling Capability and External Compensation Capability
\end{tabular}

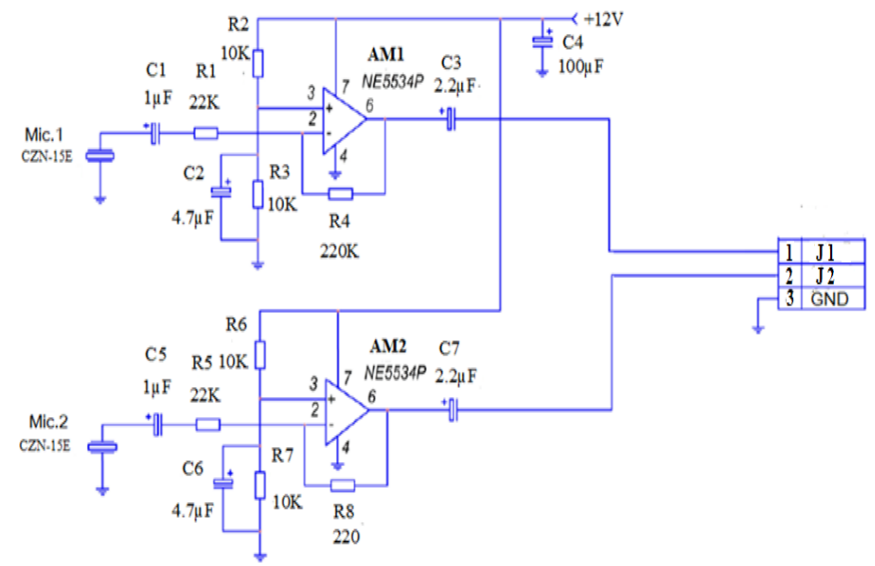

Fig. 6. The Circuit Diagrams for the Proposed Biotechnical System.

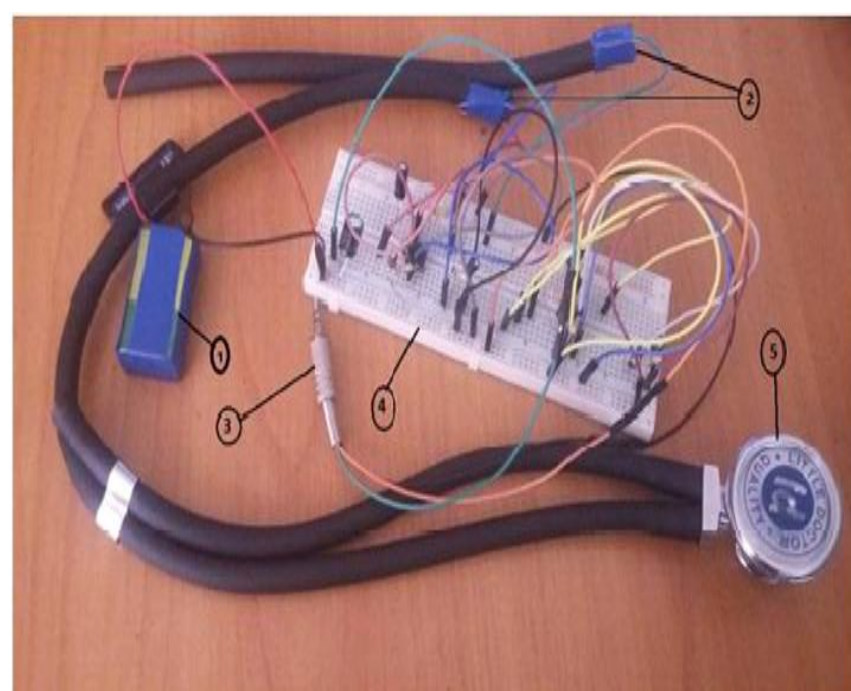

Fig. 7. Proposed Biotechnical System for Recording PCG. Numeric Indicates 1- DC Power Supply; 2- Microphone System; 3-Connector; 4- Transmitter unit; 5- Stethoscope.

The design of the biotechnical system (hardware) is shown in Fig. 7 consists of:

1) Two Electret microphones system type $\mathrm{CZN}-15 \mathrm{E})$ and two amplifiers type NE5534P

2) Transducer block and connector (3.5 jack) for transmitting the signal to the computer

3) DC Power supply (12V)

\section{B. The Description of the Software Interface}

The interface of the proposed system is shown in Fig. 8. The setup section (Fig. 9) contains parameters as follows:

- Sampling frequency (Fs) is the number of samples per second in a PCG signal [21].

- Time recoding (S) for controlling the length of the PCG signal.

- The resizing the length of the windowing filter is done by filter length button.

- In the PCG interface special button for zooming the PCG signal.

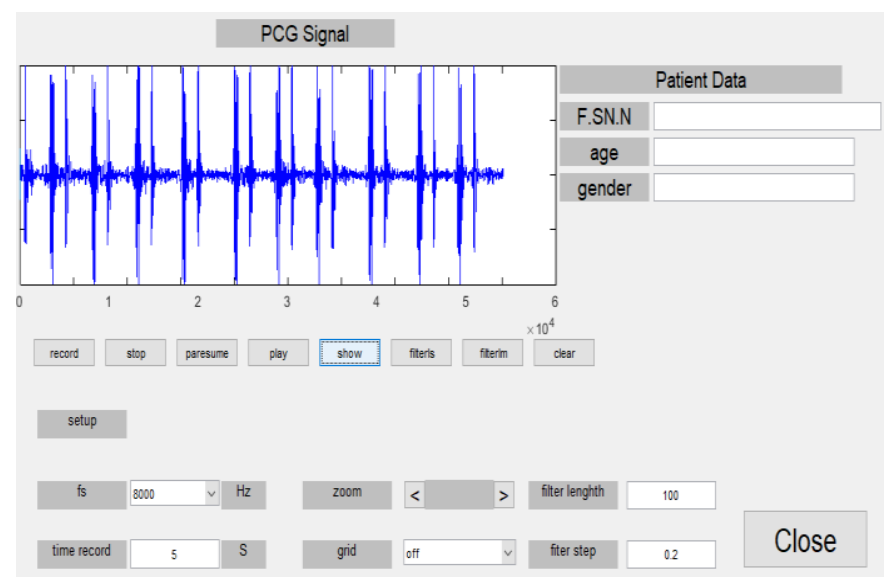

Fig. 8. Graphical user Interface of the Biotechnical. 


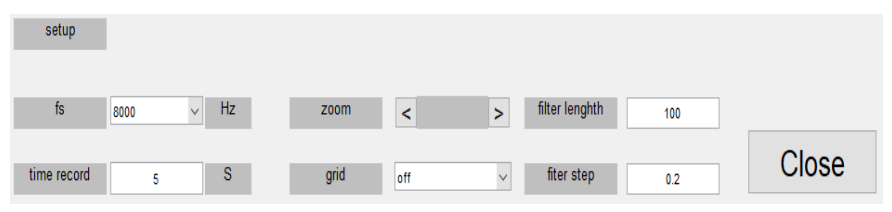

Fig. 9. Setup Section.

Fig. 10 illustrates the patient information section. In this section, the patient's metadata such as name, age, and gender can be registered and saved.

In Fig. 11 to manipulate and control the PCG signal undesired signals (noises) are expected to be recorded parallel with the PCG signal due to the physical movement of the patients or the around environment. The button of filtration contains low pass filter to remove different noises.

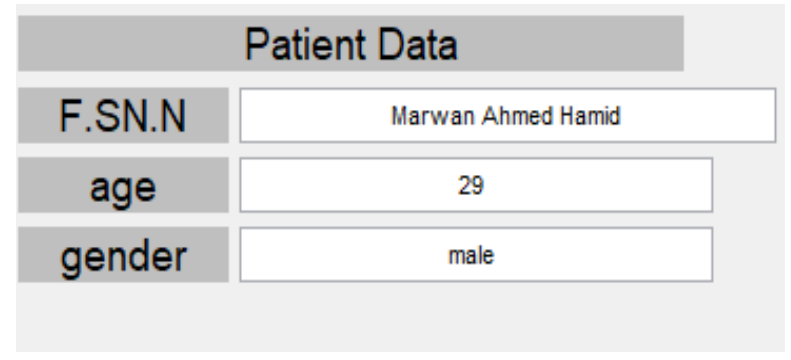

Fig. 10. Section of the Metadata.

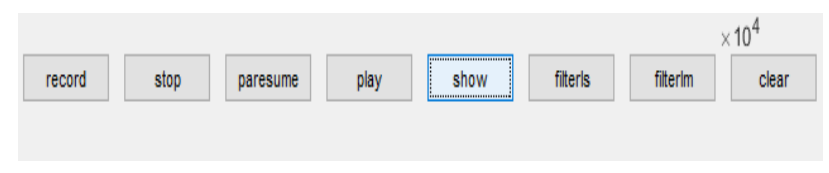

Fig. 11. Recording and Controlling Buttons.

\section{RESUlT AND DISCUSSION}

By the methodology mentioned above, the proposed biotechnical system was able to record the PCG signal (Fig. 12). The S1 and S2 peaks present clearly in the recorded PCG signal. Experimentally, it has been noticed that S1 peak values are normally larger than S2 values. Fig. 13 illustrates the time gab between $\mathrm{S} 1$ and $\mathrm{S} 2$ peaks.

The recorded PCG signal in Fig. 13 contains different type of noises [22]. In this system we used digital signal processing to denoise and enhance the quality of signal. Fig. 14 shows the PCG signal after filtering and smoothing.

The output of proposed system is plotting high fidelity recording of the heart sound. The reliability of the obtained results is verified by doctors.

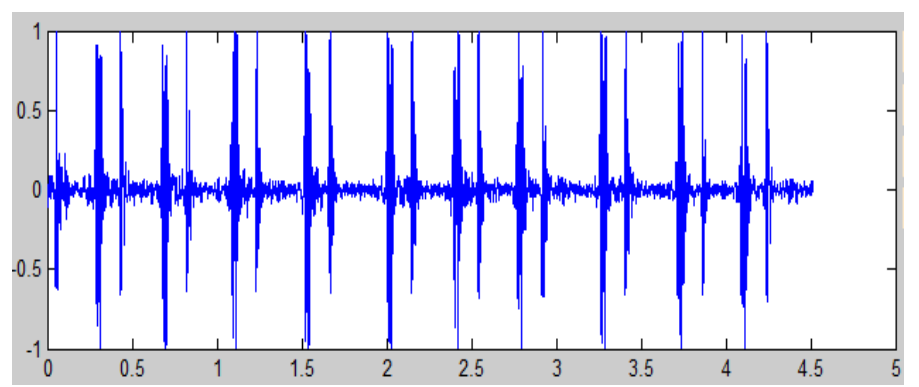

Fig. 12. Recorded PCG Signal.

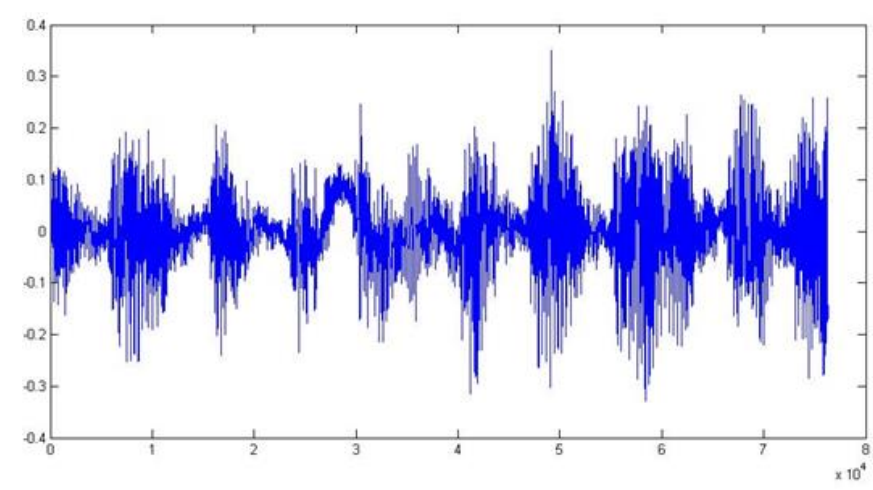

Fig. 13. S1 and S2 Peaks of the PCG Signal before Filtering and Smoothing.

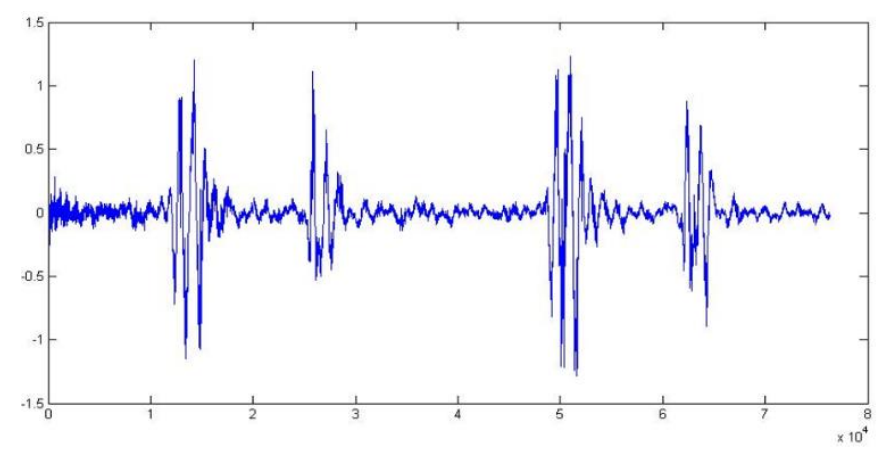

Fig. 14. PCG Signal after Denoising.

\section{CONCLUSION}

The stethoscope requires an experienced physician or doctors to analysis the heart sound. The PCG provides a useful method for studying and a diagnosis of the heart sound. By following the steps mentioned above, heart sound can be experimentally recorded and stored. The graphical user interface GUI for PCG biotechnical system (software-based system) allows easy interaction with an electrical circuit (hardware). Practically the S1 and S2 peak can be graphically detected. The confirmation of obtained results can be done by the doctors. The results (data) can be used and stored easily on a computer. The proposed method contributes to future heart sound recording research. The improvement in accuracy of the system will be better with comparative and stronger components (Hardware). The software (code) can be used in cellphones and research on signal processing methods enhances the quality of obtained PCG signal.

\section{CONFLIECT OF INTEREST}

The authors confirm that this article content has no conflict of interest.

\section{REFERENCES}

[1] Studies and E. Engineering, "Detection of Heart Diseases by Mathematical Artificial Intelligence Algorithm Using Phonocardiogram Signals," vol. 3, no. 1, pp. 145-150, 2013.

[2] P. Pcg and S. Recognition, "A thesis submitted in conformity with the requirements Graduate Department of Electrical and Computer Engineering Abstract," 2009.

[3] J. Sundberg-cohon, "The Heart: Anatomy, Physiology and Exercise Physiology,". January, 2009.

[4] L. S. Lilly, "The Cardiac Cycle: Mechanisms of Heart Sounds and Murmurs," pp. 29-45. 
[5] L. H. Cherif, "Segmentation of heart sounds and heart murmurs," January, 2016.

[6] H. Wang, Y. Fang, Z. Gao, Y. Wang, and H. You, "A Heart Sound Acquisition and Analysis System Based on PSoC4," vol. 7, no. 2, pp. 2231, 2017.

[7] D. Theodor and K. Gretzinger, "Analysis of Heart Sounds and Murmurs by Digital Signal Manipulation by 1," 1996.

[8] A. Atbi and S. M. Debbal, " Segmentation of Pathological Signals by Using Shannon Energy Envelogram," vol. 2, no. 1, pp. 1-14, 2013.

[9] T. Chowdhury, "Phonocardiography and analysis using standard deviation profile A major qualifying thesis report :," no. January, 2017.

[10] Y. M. Akay, "Detection of Coronary Occlusions Using Autoregressive Modeling of Diastolic Heart Sounds," no. May, 1990.

[11] N. Dia, J. Fontecave-jallon, P. Gumery, and B. Rivet, "Denoising Phonocardiogram signals with Non-negative Matrix Factorization informed by synchronous Electrocardiogram," pp. 51-55, 2018.

[12] R. R. Sarbandi, J. D. Doyle, M. Navidbakhsh, K. Hassani, and H. Torabiyan, "A color spectrographic phonocardiography ( CSP ) applied to the detection and characterization of heart murmurs : preliminary results," Biomed. Eng. Online, vol. 10, no. 1, p. 42, 2011.
[13] N. K. Al-qazzaz, I. F. Abdulazez, and S. A. Ridha, "Simulation Recording of an ECG , PCG , and PPG for Feature Extractions,". November, 2015.

[14] G. Son and S. Kwon, "applied sciences Classification of Heart Sound Signal Using Multiple Features," 2018.

[15] A. Mirzal, "Principles of Signal Conversion : A Brief Tutorial,". January, 2018.

[16] E. C. Microphone, "Guide for Electret Condenser Microphones."

[17] R. Article, "Short review of devices for detection of human breath sounds and heart tones," vol. 6, no. 3, 2014.

[18] J. Hillenbrand, S. Haberzettl, and G. M. Sessler, "Electret microphones with stiff diaphragms,". January, 2016.

[19] A. Equipment, C. Circuits, T. C. Amplifiers, and M. Equipment, "SE5534A Single Low Noise Operational Amplifier Pb - Free Packages are Available,"pp. 1-10, 2012.

[20] U. Guide, "Electronics Workbench TM Multisim TM 9 Simulation and Capture User Guide," . February, p. 794, 2006.

[21] A. Jeukendrup, "Heart rate monitoring: applications and limitations," no. June, 2017.

[22] C. S. Sim, J. H. Sung, S. H. Cheon, J. M. Lee, J. W. Lee, and J. Lee, "The Effects of Different Noise Types on Heart Rate Variability in Men," vol. 56, no. 1, pp. 235-243, 2015. 